Louisiana State University

LSU Digital Commons

Faculty Publications

Department of Mathematics

$12-27-2016$

\title{
New bounded backstepping control designs for time-varying systems under converging-input-converging-state conditions
}

\author{
Frederic Mazenc \\ Laboratoire des Signaux et Systèmes \\ Michael Malisoff \\ Louisiana State University \\ Jerome Weston \\ Louisiana State University
}

Follow this and additional works at: https://digitalcommons.Isu.edu/mathematics_pubs

\footnotetext{
Recommended Citation

Mazenc, F., Malisoff, M., \& Weston, J. (2016). New bounded backstepping control designs for time-varying systems under converging-input-converging-state conditions. 2016 IEEE 55th Conference on Decision and Control, CDC 2016, 3167-3171. https://doi.org/10.1109/CDC.2016.7798744
}

This Conference Proceeding is brought to you for free and open access by the Department of Mathematics at LSU Digital Commons. It has been accepted for inclusion in Faculty Publications by an authorized administrator of LSU Digital Commons. For more information, please contact ir@lsu.edu. 


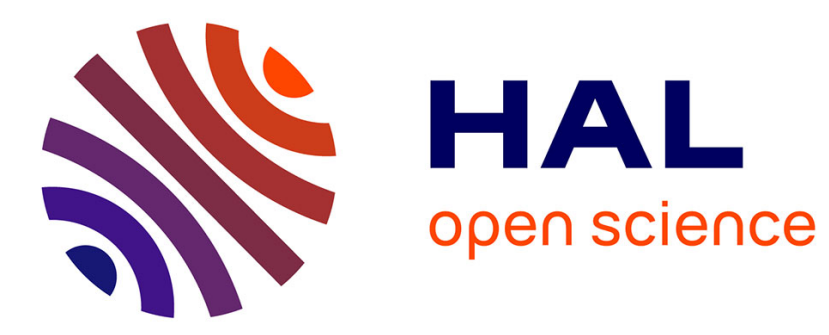

\title{
New Bounded Backstepping Control Designs for Time-Varying Systems under Converging Input Converging State Conditions
}

\author{
Frédéric Mazenc, Michael Malisoff, Jerome Weston
}

\section{- To cite this version:}

Frédéric Mazenc, Michael Malisoff, Jerome Weston. New Bounded Backstepping Control Designs for Time-Varying Systems under Converging Input Converging State Conditions. 55th IEEE Conference on Decision and Control (CDC 2016), Dec 2016, Las Vegas, United States. 10.1109/cdc.2016.7798744 . hal-01389868

\section{HAL Id: hal-01389868 \\ https://hal.inria.fr/hal-01389868}

Submitted on 30 Oct 2016

HAL is a multi-disciplinary open access archive for the deposit and dissemination of scientific research documents, whether they are published or not. The documents may come from teaching and research institutions in France or abroad, or from public or private research centers.
L'archive ouverte pluridisciplinaire HAL, est destinée au dépôt et à la diffusion de documents scientifiques de niveau recherche, publiés ou non, émanant des établissements d'enseignement et de recherche français ou étrangers, des laboratoires publics ou privés. 


\section{New Bounded Backstepping Control Designs for Time-Varying Systems under Converging Input Converging State Conditions}

Frédéric Mazenc
Michael Malisoff

Jerome Weston

\begin{abstract}
We provide new globally stabilizing backstepping controls for single input systems in a partially linear form. Instead of measuring the full state, our feedbacks use current and several time lagged values of a function of the state of the nonlinear subsystem, and have no distributed terms. Other possible advantages are that we do not require differentiability of the available nominal controls for the nonlinear subsystems, and that our controls do not contain Lie derivatives. We also allow input delays. This improves on our earlier work on the special case where the linear part has one integrator, since we now allow an arbitrary number of integrators.
\end{abstract}

Key Words: Backstepping, delays, stabilization

\section{INTRODUCTION}

This work continues our search for novel backstepping techniques that can apply in cases where the entire current state of the system may not be available for measurement; see [11], [12], [14], and [17] for some of our earlier work. Backstepping is ubiquitous in control engineering, and it usually involves recursively transforming stabilizing controllers (and corresponding Lyapunov functions) for subsystems into globally asymptotically stabilizing controllers for the entire systems; see [3], [6], [7], and [9]. However, standard backstepping generally requires measuring the entire state and leads to unbounded controls, which can be a serious limitation; see, e.g., Section VI-A below, for an example where we compare the results in this work with a standard backstepping design from [7].

One important setting where backstepping has been useful is for systems in the partial linearized single input form

$$
\left\{\begin{aligned}
\dot{x}(t) & =\mathcal{F}\left(t, x(t), z_{1}(t)\right) \\
\dot{z}_{i}(t) & =z_{i+1}(t), \quad i \in\{1,2, \ldots, k-1\} \\
\dot{z}_{k}(t) & =u(t)+h(t, x(t), z(t)) .
\end{aligned}\right.
$$

See [5, Section 9.1] for differential geometric conditions that hold for many nonlinear systems and make it possible to change variables to obtain systems of the form (1). For cases where the equations $\dot{z}_{i}(t)=z_{i+1}(t)$ are not present (which we call the $k=1$ case), our work [13] designed globally asymptotically stabilizing input delayed bounded controllers whose main advantages were that they did not involve distributed terms or Lie derivatives and did

Mazenc is with EPI DISCO Inria-Saclay, Laboratoire des Signaux et Systèmes (L2S, UMR CNRS 8506), CNRS, CentraleSupélec, Université Paris-Sud, 3 rue Joliot Curie, 91192, Gif-sur-Yvette, France. frederic.mazenc@l2s.centralesupelec.fr. Supported by l'Institut pour le Contrôle et la Décision de l'Idex Paris-Saclay (iCODE).

Malisoff and Weston are with the Department of Mathematics, 303 Lockett Hall, Louisiana State University (LSU), Baton Rouge, LA 708034918, USA. malisoffelsu.edu and jwesto3elsu.edu. Supported by NSF-ECCS Grant 1408295 . not require differentiability of the available nominal control for the $x$-subsystem. Instead, [13] used a converging input converging state condition, and then [14] gave criteria for this condition to hold. However, there is no obvious analog of [13] and [14] for the general form (1) where $k \geq 2$ is arbitrary.

This work presents a generalization of the converging input converging state assumption from [13] and [14], and uses it to design feedback controllers that drive all solutions of (1) to 0 asymptotically for any $k \geq 2$. We also present Lyapunov conditions that facilitate checking our generalized condition. We illustrate our work in two examples, including one where a scaling argument leads to a bounded backstepping controller with arbitrarily small sup norm. This contrasts with other backstepping results, such as [11] (which covers cases with only one integrator and requires certain persistency of excitation conditions that we do not use here), [12] (which uses a very different forwarding approach to cover the one integrator case), [17] (which gives unbounded controls), and the works [22], [23], and [24] (which involve Lie derivatives or measurements of the full state).

\section{Preliminaries}

We provide definitions, notation, and a key lemma. Unless otherwise noted, the dimensions of our vectors are arbitrary. The Euclidean norm of vectors in $\mathbb{R}^{a}$, and the induced norm of matrices, are denoted by $|\cdot|$. We always assume that the initial times $t_{0} \geq 0$ for all trajectories are $t_{0}=0$, but analogous results can be written for all $t_{0} \geq 0$. Given any constant $T>0$, we let $C_{\text {in }}$ denote the set of all continuous functions $\phi:[-T, 0] \rightarrow \mathbb{R}^{a}$, which we call the set of all initial functions. We define $\Xi_{t} \in C_{\text {in }}$ by $\Xi_{t}(s)=\Xi(t+s)$ for all choices of $\Xi$ and $t$ for which the equality is defined. A function $G:[0, \infty) \times \mathbb{R}^{a} \rightarrow \mathbb{R}^{p}$ is called uniformly bounded with respect to the first variable provided that there is a nondecreasing continuous function $\alpha:[0, \infty) \rightarrow[0, \infty)$ such that $|G(t, x)| \leq \alpha(|x|)$ holds for all $t \geq 0$ and $x \in \mathbb{R}^{a}$.

For each constant $r>0$, we use $\mathcal{B}_{p, r}$ to denote the closed radius $r$ ball in $\mathbb{R}^{p}$ centered at $0 \in \mathbb{R}^{p}$. We call a function $G$ : $[0, \infty) \times \mathbb{R}^{a} \rightarrow \mathbb{R}^{p}$ uniformly locally Lipschitz in the second variable provided that for each constant $K>0$, there is a constant $L_{K}>0$ such that $|G(t, x)-G(t, y)| \leq L_{K}|x-y|$ holds for all $t \geq 0$ and for all $x$ and $y$ in $\mathcal{B}_{a, K}$. Let $\mathcal{K}_{\infty}$ be the set of all continuous functions $\gamma:[0, \infty) \rightarrow[0, \infty)$ such that $\gamma(0)=0, \gamma$ is strictly increasing, and $\lim _{s \rightarrow \infty} \gamma(s)=\infty$. For any subset $S$ of a Euclidean space such that $0 \in S$, a function $\alpha: S \rightarrow[0, \infty)$ is called positive definite provided $\alpha(0)=0$ and $\alpha(r)>0$ for all $r \in S \backslash\{0\}$. A function 
$V:[0, \infty) \times \mathbb{R}^{a} \rightarrow[0, \infty)$ is called uniformly proper and positive definite provided that there exist class $\mathcal{K}_{\infty}$ functions $\underline{\alpha}$ and $\bar{\alpha}$ such that $\underline{\alpha}(|x|) \leq V(t, x) \leq \bar{\alpha}(|x|)$ hold for all $t \geq 0$ and $x \in \mathbb{R}^{a}$. We use the sup norm $|\phi|_{\mathcal{I}}=\sup _{t \in \mathcal{I}}|\phi(t)|$ for all continuous functions $\phi:[0, \infty) \rightarrow \mathbb{R}^{a}$ and all subsets $\mathcal{I} \subseteq[0, \infty)$. Throughout this work, we assume:

Assumption 1: The functions $\mathcal{F}$ and $h$ in (1) are uniformly bounded in $t$ and uniformly locally Lipschitz in $(x, z)$.

We prove the following in the appendix:

Lemma 1: Let $q>0$ be any constant. Then there are an invertible matrix $P \in \mathbb{R}^{k \times k}$ and a locally Lipschitz function $m: \mathbb{R}^{n} \times \mathbb{R}^{k} \rightarrow \mathbb{R}$ such that the change of coordinates $y=P z$ satisfies $y_{k}=z_{1}$ and transforms (1) into

$$
\left\{\begin{aligned}
\dot{x}(t) & =\mathcal{F}\left(t, x(t), y_{k}(t)\right) \\
\dot{y}_{i}(t) & =-q y_{i}(t)+y_{i-1}(t), \quad i \in\{2, \ldots, k\} \\
\dot{y}_{1}(t) & =-q y_{1}(t)+m(x(t), y(t))+u(t)
\end{aligned}\right.
$$

where $y=\left(y_{1}, \ldots, y_{k}\right)^{\top}$. If, in addition, $h=0$, then $m$ is independent of $x$ and has the form $m(y)=g_{1} y_{1}+\ldots+g_{k} y_{k}$ for some nonnegative constant $g_{i}$ 's.

The change of input $v(t)=m(x(t), y(t))+u(t)$ now gives

$$
\left\{\begin{aligned}
\dot{x}(t) & =\mathcal{F}\left(t, x(t), y_{k}(t)\right) \\
\dot{y}_{i}(t) & =-q y_{i}(t)+y_{i-1}(t), \quad i \in\{2, \ldots, k\} \\
\dot{y}_{1}(t) & =-q y_{1}(t)+v(t)
\end{aligned}\right.
$$

Hence, it suffices to build a feedback control $v(t)$ such that all solutions of (3) asymptotically converge to 0 .

Our formula for $v$ will be bounded and only depend on $k+$ 1 time lagged values of $x$, so we get a bounded backstepping theorem. Each constant $g_{i}$ from (1) will have a factor $q$, and so can be made as small as desired by reducing $q$; see the appendix. Hence, in the special key case of undelayed cases where $h=0$, our control $u$ is the sum of a bounded control term, plus a linear term with arbitrarily small coefficients.

\section{INPUT DELAYED BOUNDED BACKSTEPPING FOR (3)}

This section provides a bounded backstepping result for (3) under input delays $T \geq 0$ in the control $v$; see Section $\mathrm{V}$ where we use ideas from this section to build bounded backstepping controls for the original system (1). Assume the following (but see below for ways to check our assumptions):

Assumption 2: There are a locally Lipschitz bounded function $\omega: \mathbb{R}^{n} \rightarrow \mathbb{R}$ and constants $q>0, \tau>0$, and $T \geq 0$ such that for all continuous functions $\delta: \mathbb{R} \rightarrow \mathbb{R}$ that exponentially converge to zero, all solutions of

$$
\dot{x}(t)=\mathcal{F}\left(t, x(t), \mathcal{G}\left(x_{t}\right)+\delta(t)\right)
$$

with the choice

$$
\begin{aligned}
& \mathcal{G}\left(x_{t}\right)= \\
& \int_{t-\tau}^{t} \int_{m_{k-1}-\dot{\tau}}^{m_{k-1}} \ldots \int_{m_{1}-\tau}^{m_{1}} \frac{q^{k} e^{q\left(m_{0}-t\right)} \omega\left(x\left(m_{0}-T\right)\right)}{\left(1-e^{-q \tau}\right)^{k}} \mathrm{~d} m_{0} \ldots \mathrm{d} m_{k-1}
\end{aligned}
$$

asymptotically converge to zero. Also, $\omega(0)=0$.

We can rescale $\omega$ to replace the inner most integrand in (4) by $\omega\left(x\left(m_{0}\right)\right)$, but we use the integrand in (4), because

$$
\int_{t-\tau}^{t} \int_{m_{k-1}-\tau}^{m_{k-1}} \ldots \int_{m_{1}-\tau}^{m_{1}} \frac{q^{k} e^{q\left(m_{0}-t\right)}}{\left(1-e^{-q \tau}\right)^{k}} \mathrm{~d} m_{0} \ldots \mathrm{d} m_{k-1}=1
$$

holds for all $t \geq 0$, which will simplify our analysis below. We prove this delayed bounded backstepping result:

Theorem 1: Let Assumptions 1-2 hold. Then all solutions of (3), in closed loop with the bounded control

$$
v(t)=\frac{q^{k}}{\left(1-e^{-q \tau}\right)^{k}} \sum_{j=0}^{k} \frac{k !(-1)^{j} e^{-j q \tau}}{j !(k-j) !} \omega(x(t-j \tau-T)),
$$

asymptotically converge to 0 .

Proof: Fix any solution $(x(t), y(t))$ of (3), and set

$$
\widetilde{\omega}(x)=\frac{q^{k} \omega(x)}{\left(1-e^{-q \tau}\right)^{k}} \text { and } \tilde{\omega}^{\sharp}(r, x)=e^{r q} \tilde{\omega}(x) .
$$

Consider the operators defined by $\beta_{0}(t)=\widetilde{\omega}(x(t-T))$,

$$
\begin{aligned}
& \beta_{1}(t)=\int_{t-\tau}^{t} \widetilde{\omega}^{\sharp}\left(m_{0}-t, x\left(m_{0}-T\right)\right) \mathrm{d} m_{0}, \\
& \text { and } \beta_{j}(t)= \\
& \int_{t-\tau}^{t} \int_{m_{j-1}-\tau}^{m_{j-1}} \cdots \int_{m_{1}-\tau}^{m_{1}} \widetilde{\omega}^{\sharp}\left(m_{0}-t, x\left(m_{0}-T\right)\right) \mathrm{d} m_{0} \ldots \mathrm{d} m_{j-1}
\end{aligned}
$$

for all $j \in\{2, \ldots, k\}$, and set $b=-e^{-q \tau}$. Notice for later use that (4) reads $\dot{x}(t)=\mathcal{F}\left(t, x(t), \beta_{k}(t)+\delta(t)\right)$, and that

$$
\dot{\beta}_{j}(t)=-q \beta_{j}(t)+\beta_{j-1}(t)+b \beta_{j-1}(t-\tau), 1 \leq j \leq k
$$

The $j=1$ case of (8) follows from the Fundamental Theorem of Calculus, while for $j \geq 2$, we get

$$
\begin{aligned}
& \dot{\beta}_{j}(t)=-q \beta_{j}(t)+\beta_{j-1}(t)-e^{-q \tau} \\
& \times \int_{t-2 \tau}^{t-\tau} \ldots \int_{m_{1}-\tau}^{m_{1}} \widetilde{\omega}^{\sharp}\left(m_{0}-t+\tau, x\left(m_{0}-T\right)\right) \mathrm{d} m_{0} \ldots \mathrm{d} m_{j-2}
\end{aligned}
$$

which gives (8) for all $j \in\{1,2, \ldots, k\}$.

We next show that the operators

$$
\gamma_{k-s}(t)=\sum_{j=0}^{s} \frac{s ! b^{j}}{j !(s-j) !} \beta_{k-s}(t-j \tau)
$$

defined for all $s \in\{0, \ldots, k\}$ solve the following system:

$$
\dot{\gamma}_{i}(t)=-q \gamma_{i}(t)+\gamma_{i-1}(t), \quad i=1,2, \ldots, k
$$

To this end, notice that for all $s \in\{0,1, \ldots, k-1\}$, we can use (8) to get

$$
\begin{aligned}
\dot{\gamma}_{k-s}(t)= & \sum_{j=0}^{s} \frac{s ! b^{j}}{j !(s-j) !}\left(-q \beta_{k-s}(t-j \tau)\right. \\
& +\beta_{k-s-1}(t-j \tau) \\
& \left.+b \beta_{k-s-1}(t-(j+1) \tau)\right) \\
= & -q \gamma_{k-s}(t)+\sum_{j=0}^{s} \frac{s ! b^{j} \beta_{k-s-1}(t-j \tau)}{j !(s-j) !} \\
& +\sum_{j=1}^{s+1} \frac{s ! b^{j} \beta_{k-s-1}(t-j \tau)}{(j-1) !(s-j+1) !} .
\end{aligned}
$$

For each $j \in\{1, \ldots, s\}$, the coefficient of $b^{j} \beta_{k-s-1}(t-j \tau)$ in the summation terms in (11) add to

$$
\frac{s !}{j !(s-j) !}+\frac{s !}{(j-1) !(s-j+1) !}=s ! \frac{s-j+1+j}{j !(s-j+1) !}=\frac{(s+1) !}{j !(s+1-j) !},
$$

and the coefficients of $\beta_{k-s-1}(t)$ and $b^{s+1} \beta_{k-s-1}(t-(s+$ $1) \tau)$ in (11) are both 1 , which gives (10).

Next, let $\xi_{i}(t)=y_{i}(t)-\gamma_{i}(t)$ for all $i \in\{1,2, \ldots, k\}$. Then we can combine (3) with (10) and then use the fact 
that our controller $v(t)$ in (6) is $\gamma_{0}(t)$ to obtain this system:

$$
\left\{\begin{aligned}
\dot{x}(t) & =\mathcal{F}\left(t, x(t), \xi_{k}(t)+\gamma_{k}(t)\right) \\
\dot{\xi}_{i}(t) & =-q \xi_{i}(t)+\xi_{i-1}(t), \quad i \in\{2, \ldots, k\} \\
\dot{\xi}_{1}(t) & =-q \xi_{1}(t)
\end{aligned}\right.
$$

The $\xi$-subsystem of (12) is globally exponentially stable to zero, and we can use a corresponding quadratic Lyapunov function for this system (e.g., $V(\xi)=c_{0} \xi_{k}^{2}+\ldots+c_{k-1} \xi_{1}^{2}$, where $c_{0}=1$ and $c_{i}=\left(c_{i-1}^{2} /\left(2 q^{2}\right)\right)+1$ for $i=1,2, \ldots, k-$ 1 ) to prove input-to-state stability (or ISS) for (10) with respect to $\gamma_{0}(t)$; see [7] for background on ISS. This leads us to consider $\dot{x}(t)=\mathcal{F}\left(t, x(t), \gamma_{k}(t)+\delta(t)\right)$, where $\delta=\xi_{k}$ exponentially converges to zero. Since $\gamma_{k}=\beta_{k}$, Assumption 2 now gives $\lim _{t \rightarrow \infty} x(t)=0$, so $\lim _{t \rightarrow \infty} \gamma_{0}(t)=0$, because $\omega(0)=0$. Since (10) satisfies ISS with respect to $\gamma_{0}(t)$, it follows that $\lim _{t \rightarrow \infty} \gamma_{i}(t)=0$ for all $i \in\{1,2, \ldots, k\}$, so $\lim _{t \rightarrow \infty} y_{i}(t)=\lim _{t \rightarrow \infty} \xi_{i}(t)+\lim _{t \rightarrow \infty} \gamma_{i}(t)=0$ for all $i \in\{1,2, \ldots, k\}$, which proves Theorem 1 .

\section{Ways to Check Assumption 2}

As in the special case where $k=1$ that we studied in [14], a key feature of the preceding result for general $k>1$ is that we do not require the nominal control $\omega$ to render

$$
\dot{x}(t)=\mathcal{F}(t, x(t), \omega(x(t)))
$$

uniformly globally asymptotically stable to 0 , nor do we require differentiability of $\omega$ or Lyapunov functions for the $x$ dynamics. However, we can use (13) to give sufficient conditions for Assumption 2 to hold. One way to do so is as follows, where $V_{t}$ and $V_{x}$ denote the partial derivative with respect to $t$ and the gradient with respect to $x$, respectively:

Assumption 3: The function $\mathcal{F}$ admits the control affine form $\mathcal{F}(t, x, p)=f(t, x)+g(t, x) p$ where $f:[0, \infty) \times$ $\mathbb{R}^{n} \rightarrow \mathbb{R}^{n}$ and $g:[0, \infty) \times \mathbb{R}^{n} \rightarrow \mathbb{R}^{n}$ are continuous and uniformly locally Lipschitz in the second variable. Also, there are a $C^{1}$ uniformly proper and positive definite function $V:[0, \infty) \times \mathbb{R}^{n} \rightarrow[0, \infty) ;$ a uniformly continuous positive definite function $W: \mathbb{R}^{n} \rightarrow[0, \infty)$; positive constants $r_{0}$, $r_{1}$, and $r_{3}$; and a constant $r_{2} \geq 0$ such that

$$
\begin{aligned}
& V_{t}(t, x)+V_{x}(t, x)(f(t, x)+g(t, x) \omega(x)) \leq-W(x), \\
& \left|V_{x}(t, x) g(t, x)\right| \leq r_{0} \sqrt{W(x)},|\omega(x)| \leq r_{1} \sqrt{W(x)}, \\
& |f(t, x)| \leq r_{2} \sqrt{W(x)}, \text { and }|g(t, x)| \leq r_{3}
\end{aligned}
$$

hold for all $(t, x) \in[0, \infty) \times \mathbb{R}^{n}$, where $\omega: \mathbb{R}^{n} \rightarrow \mathbb{R}$ is bounded, satisfies $\omega(0)=0$, and has a global Lipschitz constant $C>0$.

See [15] for general conditions under which Assumption 3 holds. Fix any constants $\tau>0$ and $T \geq 0$. Set $k^{\sharp}=k \tau+T$ and

$$
\begin{aligned}
& \mathcal{M}_{*}= \\
& 4\left(C r_{0} k^{\sharp}\right)^{2}\left\{2 r_{2}^{2}+\frac{5}{2}\left(k^{\sharp} r_{1} r_{3}\right)^{2} \tau^{2(k-1)}\left(\frac{q}{1-e^{-q \tau}}\right)^{2 k}\right\} .
\end{aligned}
$$

When $T=0$, we get $\mathcal{M}_{*} \rightarrow 0$ as $\tau \rightarrow 0^{+}$, in which case we can satisfy the condition $\mathcal{M}_{*}<1$ from the following proposition for any values of the other constants, by choosing $\tau>0$ small enough:
Proposition 1: Let Assumption 3 hold and $\mathcal{M}_{*}<1$. Then for all continuous functions $\delta:[0, \infty) \rightarrow \mathbb{R}$ that exponentially converge to 0 , all solutions of (4) asymptotically converge to zero, so Assumption 2 is satisfied.

Proof: Along all trajectories of (4) for large enough $t$, the functions $V$ and $W$ from Assumption 3 satisfy

$$
\begin{aligned}
& \dot{V} \leq-W(x(t))+V_{x}(t, x(t)) g(t, x(t))[\delta(t)+ \\
& \left.\quad \int_{t-\tau}^{t} \int_{m_{k-1}-\tau}^{m_{k-1}} \cdots \int_{m_{1}-\tau}^{m_{1}} \delta \tilde{\omega}\left(m_{0}, t\right) \mathrm{d} m_{0} \ldots \mathrm{d} m_{k-1}\right] \\
& \leq-W(x(t))+r_{0} \sqrt{W(x(t))}\left(C \int_{t-k^{\sharp}}^{t}|\dot{x}(\ell)| \mathrm{d} \ell+|\delta(t)|\right),
\end{aligned}
$$

where $\delta \tilde{\omega}\left(m_{0}, t\right)=e^{q\left(m_{0}-t\right)}\left(\tilde{\omega}\left(x\left(m_{0}-T\right)\right)-\tilde{\omega}(x(t))\right)$ and $\tilde{\omega}$ is from (7), and where we used the relations (5) and

$$
\begin{aligned}
& \sup _{m_{0} \in\left[t-k^{\sharp}, t\right]}\left|\omega\left(x\left(m_{0}\right)\right)-\omega(x(t))\right| \\
& \leq C \sup _{m_{0} \in\left[t-k^{\sharp}, t\right]}\left|x\left(m_{0}\right)-x(t)\right| \leq C \int_{t-k^{\sharp}}^{t}|\dot{x}(\ell)| \mathrm{d} \ell .
\end{aligned}
$$

Hence, Jensen's inequality gives

$$
\begin{aligned}
\dot{V} \leq & -\frac{1}{2} W(x(t))+r_{0}^{2}|\delta(t)|^{2} \\
& +C^{2} r_{0}^{2} k^{\sharp} \int_{t-k^{\sharp}}^{t}|\dot{x}(\ell)|^{2} \mathrm{~d} \ell,
\end{aligned}
$$

where we also used Young's Inequality to get $b \sqrt{W(x(t))} \leq$ $0.25 W(x(t))+b^{2}$ twice for suitable choices of $b \geq 0$.

We can also use the Young and Jensen inequalities, and the fact that $(a+b)^{2} \leq(5 / 4) a^{2}+5 b^{2}$ holds for all $a \geq 0$ and $b \geq 0$, to upper bound the integrand in (17) by

$$
\begin{aligned}
|\dot{x}(\ell)|^{2} \leq & 2|f(\ell, x(\ell))|^{2}+2|g(\ell, x(\ell))|^{2}(|\delta(\ell)|+ \\
& \left.\tau^{k-1}\left(\frac{q}{1-e^{-q \tau}}\right)^{k} \int_{\ell-k^{\sharp}}^{\ell}\left|\omega\left(x\left(m_{0}\right)\right)\right| \mathrm{d} m_{0}\right)^{2} \\
\leq & 2 r_{2}^{2} W(x(\ell))+2 r_{3}^{2}\left(5|\delta(\ell)|^{2}+\right. \\
& \left.\frac{5 k^{\sharp}}{4 \tau}\left(\frac{\tau q}{1-e^{-q \tau}}\right)^{2 k} \int_{\ell-k^{\sharp}}^{\ell} \omega^{2}\left(x\left(m_{0}\right)\right) \mathrm{d} m_{0}\right) \\
\leq & 2 r_{2}^{2} W(x(\ell))+10 r_{3}^{2}|\delta(\ell)|^{2}+ \\
& \frac{5 k^{\sharp} r_{1}^{2} r_{3}^{2}}{2 \tau}\left(\frac{\tau q}{1-e^{-q \tau}}\right)^{2 k} \int_{\ell-k^{\sharp}}^{\ell} W\left(x\left(m_{0}\right)\right) \mathrm{d} m_{0} .
\end{aligned}
$$

Combining the preceding bound with (17) gives the estimate

$$
\begin{aligned}
\dot{V} \leq & -\frac{1}{2} W(x(t))+\mathcal{M}_{1} \int_{t-2 k \tau}^{t} W(x(\ell)) \mathrm{d} \ell \\
& +\mathcal{M}_{2}|\delta|_{\left[t-k^{\sharp}, t\right]}^{2}, \text { where }
\end{aligned}
$$

$\mathcal{M}_{1}=\left(r_{0} C\right)^{2} k^{\sharp}\left\{2 r_{2}^{2}+\frac{5}{2}\left(k^{\sharp} r_{1} r_{3}\right)^{2} \tau^{2(k-1)}\left(\frac{q}{1-e^{-q \tau}}\right)^{2 k}\right\}$

and $\mathcal{M}_{2}=r_{0}^{2}+10\left(C r_{0} r_{3} k^{\sharp}\right)^{2}$.

Also, our bound $\mathcal{M}_{*}<1$ can be written as $4 k^{\sharp} \mathcal{M}_{1}<1$. Hence, we can find a constant $\lambda>1$ such that $2 \lambda k^{\sharp} \mathcal{M}_{1}<\frac{1}{2}$. Then along all trajectories of (4), the function

$$
V_{1}\left(t, x_{t}\right)=V(t, x(t))+\lambda \mathcal{M}_{1} \int_{t-2 k^{\sharp}}^{t} \int_{s}^{t} W(x(\ell)) \mathrm{d} \ell \mathrm{d} s
$$

satisfies

$$
\begin{aligned}
\dot{V}_{1} \leq & -\left\{\frac{1}{2}-2 \lambda k^{\sharp} \mathcal{M}_{1}\right\} W(x(t))+\mathcal{M}_{2}|\delta|_{\left[t-k^{\sharp}, t\right]}^{2} \\
& +\mathcal{M}_{1}(1-\lambda) \int_{t-2 k^{\sharp}}^{t} W(x(\ell)) \mathrm{d} \ell \\
\leq & -\left\{\frac{1}{2}-2 \lambda k^{\sharp} \mathcal{M}_{1}\right\} W(x(t))+\mathcal{M}_{2}|\delta|_{\left[t-k^{\sharp}, t\right]}^{2} .
\end{aligned}
$$


Since the quantity in curly braces in (19) is positive and $\delta$ exponentially converges to 0 , we can integrate (19) to conclude that $V_{1}\left(t, x_{t}\right)$ is bounded. Since $V$ is uniformly proper and positive definite, it follows that $|x(t)|$ is bounded. It follows from the structure of the $x(t)$ dynamics that $x(t)$ is uniformly continuous. Recalling that $W$ is uniformly continuous, we conclude that $W(x(t))$ is a uniformly continuous function of $t$. Also, since $\delta \in \mathcal{L}_{2}([0, \infty), \mathbb{R})$, the inequality (19) gives $\int_{0}^{\infty} W(x(\ell)) \mathrm{d} \ell<\infty$. It follows from Barbalat's Lemma that $\lim _{t \rightarrow \infty} W(x(t))=0$, so since $W$ is positive definite, we get $\lim _{t \rightarrow \infty} x(t)=0$, as needed.

\section{Bounded BACKSTEPPING CONTROLLERS FOR (1)}

\section{A. Undelayed Case}

A potential advantage of the control $v(t)$ from Theorem 1 is its simple form, which leads to the control $u(t)=$ $v(t)-g P z(t)$ for the original system (1) when $h=0$ case, where $P$ and $g=\left(g_{1}, \ldots, g_{k}\right)$ are from Lemma 1 . When $h$ is an arbitrary bounded locally Lipschitz function, we can use an analog of Theorem 1 to find globally bounded (but more complicated) controllers $u$ such that all solutions of (1) asymptotically converge to 0 , as follows. We assume that we know a constant $\tau>0$ and a bounded globally Lipschitz function $\omega: \mathbb{R}^{n} \rightarrow \mathbb{R}$ such that $\omega(0)=0$ and such that for each continuous function $\delta:[0, \infty) \rightarrow \mathbb{R}$ that exponentially converges to 0 , all solutions of $\dot{x}(t)=$ $\mathcal{F}\left(t, x(t), \alpha_{k}\left(x_{t}\right)+\delta(t)\right)$ asymptotically converge to 0 , where

$$
\begin{aligned}
& \alpha_{k}\left(x_{t}\right)= \\
& \frac{1}{\tau^{k}} \int_{t-\tau}^{t} \int_{m_{k-1}-\tau}^{m_{k-1}} \cdots \int_{m_{1}-\tau}^{m_{1}} \omega\left(x\left(m_{0}\right)\right) \mathrm{d} m_{0} \ldots \mathrm{d} m_{k-1} .
\end{aligned}
$$

We can provide sufficient conditions for the preceding assumption to hold, using a variant of Proposition 1.

Then we set $\tilde{z}_{i}=z_{i}-\beta_{i}$ for all $i \in\{1,2, \ldots, k\}$, where $\beta_{i}(t)=D_{t}^{(i-1)} \alpha_{k}\left(x_{t}\right)$ is the $(i-1)$-st derivative of $\alpha_{k}\left(x_{t}\right)$ in $t$, so $\beta_{1}(t)=\alpha_{k}\left(x_{t}\right)$. This produces the system

$$
\left\{\begin{aligned}
\dot{x}(t) & =\mathcal{F}\left(t, x(t), \beta_{1}(t)+\tilde{z}_{1}(t)\right) \\
\tilde{\tilde{z}}_{i}(t) & =\tilde{z}_{i+1}(t), \quad i \in\{1,2, \ldots, k-1\} \\
\dot{\tilde{z}}_{k}(t) & =u(t)+h(t, x(t), z(t))-\dot{\beta}_{k}(t) .
\end{aligned}\right.
$$

Also, results from [21] provide a nested saturation bounded feedback $\mu$ such that the $k$-dimensional system

$$
\dot{a}_{i}=a_{i+1} \text { for } 1 \leq i \leq k-1, \quad \dot{a}_{k}=\mu(a)
$$

is globally asymptotically and locally exponentially stable to 0 . Hence, using the bounded control $u(t)=$ $\mu(\tilde{z}(t))-h(t, x(t), z(t))+\dot{\beta}_{k}(t)$ in (21) produces an exponentially stable $\tilde{z}$ subsystem, so our assumption ensures that $\lim _{t \rightarrow \infty} x(t)=0$, so $\lim _{t \rightarrow \infty} \beta_{i}(t)=0$ holds for all $i$ (e.g., by the Dominated Convergence Theorem), which gives $\lim _{t \rightarrow \infty} z_{i}(t)=\lim _{t \rightarrow \infty} \tilde{z}_{i}(t)+\lim _{t \rightarrow \infty} \beta_{i}(t)=0$ for all $i$.

\section{B. Input Delayed Cases}

By replacing the integrand $\omega\left(x\left(m_{0}\right)\right)$ in the definition (20) of $\alpha_{k}$ by $\omega\left(x\left(m_{0}-T\right)\right)$, we can generalize the preceding bounded backstepping result to cover the systems (1) with $h=0$ and any input delay $T \geq 0$. This extends the delayed bounded backstepping results from [14] (which were confined to the $k=1$ case) to cover any choice $k \geq 2$ of the dimension of $z$. To see how this extension can be done, first note that for any constant delay $T \geq 0$, we can find a bounded nested saturation controller $\mu$ such that

$$
\dot{a}_{i}(t)=a_{i+1}(t) \text { for } 1 \leq i \leq k-1, \quad \dot{a}_{k}(t)=\mu(t-T)
$$

is globally asymptotically and locally exponentially stable to 0 . This is done by using a time rescaling $t=v s$ for a suitable constant $v>0$, the changes of variables $\tilde{a}_{i}(s)=v^{i-1} a_{i}(v s)$, and the new control $\tilde{\mu}(s-T / v)=v^{k} \mu(v s-T)$ to get

$$
\left\{\begin{array}{l}
\dot{\tilde{a}}_{i}(s)=\tilde{a}_{i+1}(s), \quad 1 \leq i \leq k-1 \\
\dot{\tilde{a}}_{k}(s)=\tilde{\mu}(s-T / v),
\end{array}\right.
$$

and then using the nested saturation construction from [16, Theorem 8] to get a globally asymptotically and locally exponentially stabilizing controller $\tilde{\mu}$ for (24) when $v$ is large enough. This implies the desired asymptotic properties of (23), and then we choose $u(t-T)=\mu(t-T)+\dot{\beta}_{k}(t)$ to show the stability properties as before, where $\mu(t)=\tilde{\mu}(t / v) / v^{k}$. This is a valid choice of the control, because our replacement in the integrand for $\alpha_{k}$ implies that $\dot{\beta}_{k}(t)$ only depends on $x$ values on $(-\infty, t-T]$.

\section{EXAMPLES}

\section{A. Comparison with Standard Backstepping}

We can sometimes apply Theorem 1 by checking Assumption 2 through a mixture of Lyapunov and direct trajectory analyses. For instance, consider the three dimensional system

$$
\left\{\begin{array}{l}
\dot{x}=x^{2}-x^{3}+z_{1} \\
\dot{z}_{1}=z_{2}, \quad \dot{z}_{2}=u .
\end{array}\right.
$$

As noted in [7, pages 593-594], the system (25) is globally asymptotically stabilized by the unbounded control

$$
\begin{aligned}
& u(x, z)=-\frac{\partial V_{0}}{\partial z_{1}}\left(x, z_{1}\right)+\frac{\partial \phi}{\partial z_{1}}\left(x, z_{1}\right) z_{2}-z_{2} \\
& +\frac{\partial \phi}{\partial x}\left(x, z_{1}\right)\left(x^{2}-x^{3}+z_{1}\right)+\phi\left(x, z_{1}\right), \\
& \text { where } V_{0}\left(x, z_{1}\right)=\frac{1}{2} x^{2}+\frac{1}{2}\left(z_{1}+x+x^{2}\right)^{2} \text { and } \\
& \phi\left(x, z_{1}\right)=-x-(1+2 x)\left(x^{2}-x^{3}+z_{1}\right) \\
& -\left(z_{1}+x+x^{2}\right) \text {. }
\end{aligned}
$$

By contrast, we can build our control from Theorem 1 for (25), by checking that Assumption 2 holds with $\omega(x)=$ $-\sin (\pi x / 2) \mathbf{1}_{[-2,2]}(x)$, where the indicator function $\mathbf{1}_{[-2,2]}$ is defined to be 1 on $[-2,2]$ and 0 on $\mathbb{R} \backslash[-2,2]$.

To verify Assumption 2 for this choice of $\omega$, first note that for each continuous function $\delta: \mathbb{R} \rightarrow \mathbb{R}$ that exponentially converges to 0 and each initial state $x_{0} \in \mathbb{R}$, we can use (5) with $k=2$ to find a value $T\left(x_{0}, \delta\right) \in[0, \infty)$ such that for all constants $q>0$ and $\tau>0$, the corresponding solution of

$$
\begin{aligned}
& \dot{x}(t)=x^{2}(t)-x^{3}(t)+ \\
& \int_{t-\tau}^{t} \int_{m_{1}-\tau}^{m_{1}} \frac{q^{2} e^{q\left(m_{0}-t\right)}}{\left(1-e^{-q \tau}\right)^{2}} \omega\left(x\left(m_{0}\right)\right) \mathrm{d} m_{0} \mathrm{~d} m_{1}+\delta(t)
\end{aligned}
$$

satisfies $x(t) \in[-0.8,3 / 2]$ for all $t \geq T\left(x_{0}, \delta\right)$. This can be done by noting that the integral in (26) is bounded by 1 , that $x^{2}-x^{3} \leq-1.125$ for all $x \geq 3 / 2$, and that $x^{2}-x^{3} \geq 1.152$ for all $x \leq-0.8$, so the right side terms $x^{2}(t)-x^{3}(t)$ in 

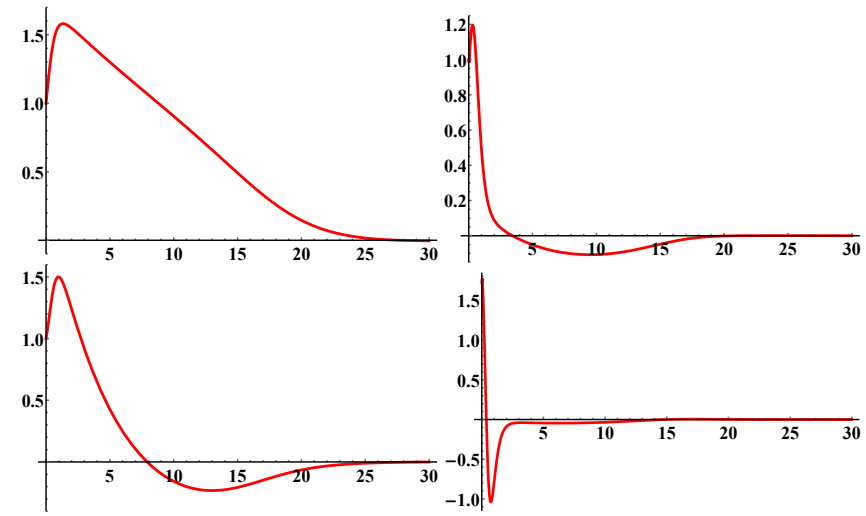

Fig. 1. Simulating System (25) with Control (27), $q=0.345$, and $\tau=$ 0.029. All Initial States Set to 1. Upper Left: $x(t)$. Upper Right: $z_{1}(t)$. Lower Left: $z_{2}(t)$. Lower Right: $u(t)$.

(26) dominate the other right side terms. Hence, it suffices to check the inequalities (14) from Assumption 3 for all $x \in$ $[-0.8,3 / 2]$, by only considering values of $t \geq T\left(x_{0}, \delta\right)$.

We now check the estimates from (14) for all $x \in$ $[-0.8,3 / 2]$ using $V(x)=\frac{1}{2} x^{2}, W(x)=x^{2}, f(x)=x^{2}-x^{3}$, and $g(x)=1$. First note that simple calculations (e.g., using Mathematica [10]) give $x^{2}-x^{3}-\sin (\pi x / 2) \leq-x$ (resp., $\geq-x$ ) for all $x \in[0,3 / 2]$ (resp., $x \in[-0.8,0]$ ) which gives $\nabla V(x)(f(x)+\omega(x)) \leq-W(x),\left|x^{2}-x^{3}\right| \leq 1.44|x|$, and $|\sin (\pi x / 2)| \leq(\pi / 2)|x|$ when $x \in[-0.8,3 / 2]$, so we can choose $r_{0}=1, r_{1}=\pi / 2, r_{2}=1.44, r_{3}=1$, and $C=\pi / 2$. Hence, the sufficient condition $\mathcal{M}_{*}<1$ from Proposition 1 (for $\lim _{t \rightarrow \infty} x(t)=0$ to hold) is satisfied by the choices $q=0.345$ and $\tau=0.029$, since these choices give

$$
4\left(\frac{\pi}{2}\right)^{2} 4 \tau^{2}\left\{2(1.44)^{2}+\frac{5}{2}(4)\left(\frac{\tau q}{1-e^{-q \tau}}\right)^{4} \frac{\pi^{2}}{4}\right\} \approx 0.97<1 .
$$

Using the change of feedback that transformed (2) into (3) and the formula for the function $m$ in (3) in the appendix, we obtain the stabilizing controller

$$
\begin{aligned}
& u(t)=\frac{q^{2}}{\left(1-e^{-q \tau}\right)^{2}}\left\{\omega(x(t))-2 e^{-q \tau} \omega(x(t-\tau))+\right. \\
& \left.e^{-2 q \tau} \omega(x(t-2 \tau))\right\}-2 q z_{2}(t)-q^{2} z_{1}(t), \\
& \text { where } \omega(x)=-\sin \left(\frac{\pi x}{2}\right) \mathbf{1}_{[-2,2]}(x) .
\end{aligned}
$$

To illustrate our results, we used Mathematica to simulate (25) in closed loop with (27) and the preceding parameter values, with the initial states $x=z_{1}=z_{2}=1$, assuming that $x$ takes the constant value 1 at negative times in order to initialize the controller, and we plotted our results in Figure 1. They illustrate the rapid convergence of the states to 0 .

\section{B. Controls of Arbitrarily Small Amplitude}

Consider the special case

$$
\left\{\begin{aligned}
\dot{x}(t) & =y_{2}(t) \\
\dot{y}_{2}(t) & =-q y_{2}+y_{1}(t) \\
\dot{y}_{1}(t) & =-q y_{1}+v(t)
\end{aligned}\right.
$$

of (3) and the function $\omega(x)=-\varepsilon \operatorname{sat}(x)$, where sat is the usual saturation that is defined by $\operatorname{sat}(x)=x$ if $|x| \leq 1$ and $\operatorname{sat}(x)=\operatorname{sign}(x)$ if $|x|>1$, and where $\varepsilon>0$ is

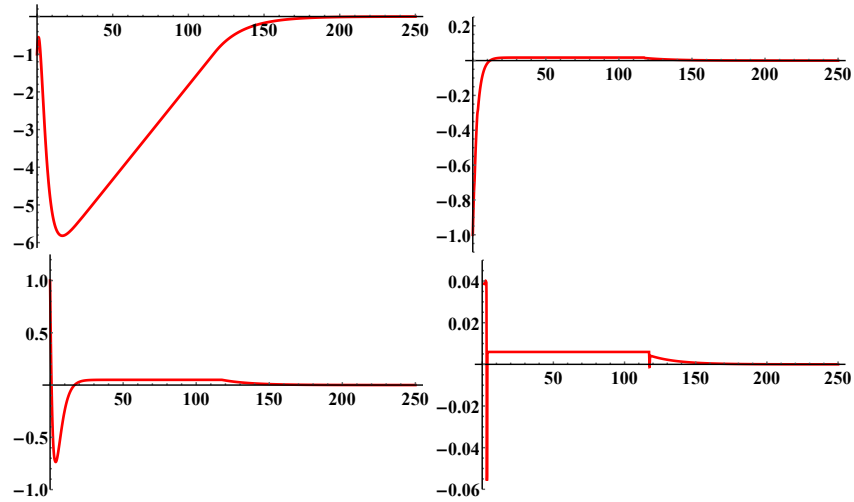

Fig. 2. Simulating (28) with Control (30), $\tau=0.35, q=0.345, \varepsilon=0.05$, and $T=0.7$. Initial State Vector: $(-1,-1,1)$. Upper Left: $x(t)$. Upper Right: $y_{1}(t)$. Lower Left: $y_{2}(t)$. Lower Right: $v(t)$.

a constant. The assumptions of Proposition 1 are satisfied, by choosing $k=2, \mathcal{F}(t, x, p)=p, V(x)=\int_{0}^{x} \operatorname{sat}(s) \mathrm{d} s$, $W(x)=\varepsilon_{\operatorname{sat}^{2}}(x), C=\varepsilon, r_{0}=1 / \sqrt{\varepsilon}, r_{1}=\sqrt{\varepsilon}, r_{2}=0$, and $r_{3}=1$ in Assumption 2, and any choices of the constants $\varepsilon>0, \tau>0$, and $q>0$ and the delay $T \geq 0$ such that

$$
\sqrt{10} \tau \varepsilon(2 \tau+T)^{2}\left(\frac{q}{1-e^{-q \tau}}\right)^{2}<1 .
$$

Then our theorem ensures that all solutions of (28) in closed loop with the control

$$
\begin{aligned}
& v(t)=-\frac{q^{2} \varepsilon}{\left(1-e^{-q \tau}\right)^{2}}\{\operatorname{sat}(x(t-T))- \\
& \left.2 e^{-q \tau} \operatorname{sat}(x(t-\tau-T))+e^{-2 q \tau} \operatorname{sat}(x(t-2 \tau-T))\right\}
\end{aligned}
$$

from (6) asymptotically converge to 0 . We can allow controls of arbitrarily small sup norm, and any delay $T \geq 0$, by choosing $\varepsilon>0$ small enough. Also, only the value of $\operatorname{sat}(x)$ is needed. To illustrate our control (30), we used Mathematica to simulate (28) in closed loop with (30) with the initial state vector $(-1,-1,1)$ the parameter choices $\tau=0.35, q=0.345, \varepsilon=0.05$, and $T=0.7$, which satisfy (29), and we report our results in Figure 2. They show convergence of the states and control values to 0 .

\section{Normal Forms for Underactuated Systems}

Normal form systems (corresponding to (1) with $h=0$ ) commonly arise when modeling two degree-of-freedom (or DOF) underactuated systems. For instance, [19, Chapter 3] shows that the acrobot, inertia-wheeled pendulum, and translational oscillation with an eccentric rotational actuator (or TORA) models admit changes of coordinates that produce

$$
\left\{\begin{array}{l}
\dot{x}_{1}=m_{11}^{-1}\left(z_{1}\right) x_{2}, \quad \dot{x}_{2}=g_{1}\left(x_{1}-\gamma\left(z_{1}\right), z_{1}\right), \\
\dot{z}_{1}=z_{2}, \quad \dot{z}_{2}=u
\end{array}\right.
$$

where $M=\left[m_{i j}\right] \in \mathbb{R}^{2 \times 2}$ is the inertia matrix, $g_{1}(x, z)=$ $-\partial V / \partial x, V$ is the potential energy, and

$$
\gamma\left(z_{1}\right)=\int_{0}^{z_{1}} m_{11}^{-1}(\theta) m_{12}(\theta) \mathrm{d} \theta
$$

Our dynamics (1) also cover the planar vertical takeoff and landing (or PVTOL) model [4], [20] after suitable changes of coordinates. Due to space constraints, we will not discuss 
these examples here, but it is worth studying how our novel control designs could also benefit the study of these systems.

\section{CONCLUSIONS}

We introduced a key extension of our converging input converging state assumption from [13], which allowed us to extend the bounded backstepping results from [14] for systems with one integrator to higher dimensional partially linear systems with an arbitrary number of integrators. We gave Lyapunov-like sufficient conditions for our new assumption to hold. Our control formulas do not require any Lie derivatives, nor do they require any differentiability of the nominal control for the nonlinear subsystems. Our main controls ensure globally asymptotic stability to the equilibrium, and are a sum of (i) a bounded output term involving the state of the nonlinear subsystem and (ii) a linear combination of the states of the linearized part that can be scaled by an arbitrarily small constant. We also covered input delays, using a nested saturation approach that produced globally asymptotically stabilizing bounded controls. We plan to apply our work to the PVTOL and other engineering systems that can be put in triangular form, e.g., by combining our methods with ideas from [1], [2], and [8].

\section{APPENDIX}

We prove Lemma 1 . Set $y_{k}=z_{1}$. For all $i \in\{1,2, \ldots, k-$ $1\}$, we recursively construct linear functions of the form

$$
\mathcal{L}_{i}\left(z_{1}, \ldots, z_{i}\right)=\sum_{j=1}^{i} r_{i j} z_{j}
$$

for some constants $r_{i j}>0$, and functions $y_{k-i}$ of $z$, such that these two equalities hold:

$$
y_{k-i}=\dot{y}_{k-i+1}+q y_{k-i+1}=z_{i+1}+q \mathcal{L}_{i}\left(Z_{i}\right),
$$

where $Z_{i}=\left(z_{1}, z_{2}, \ldots, z_{i}\right)$. For $i=1$, our condition $y_{k}=$ $z_{1}$ allows us to write (A.2) as $y_{k-1}=z_{2}+q z_{1}=z_{2}+$ $q \mathcal{L}_{1}\left(z_{1}\right)$, so we can take $y_{k-1}=z_{2}+q z_{1}$ and $\mathcal{L}_{1}\left(z_{1}\right)=z_{1}$. Assume that we found $\mathcal{L}_{i}$ and $y_{k-i}$ satisfying (A.2) for all $i \in\{1, \ldots, \ell\}$ for some $\ell<k-1$. We show how to satisfy (A.2) for $i=\ell+1$. Our requirement (A.2) for $i=\ell+1$ is

$$
\begin{aligned}
y_{k-\ell-1}=\dot{y}_{k-\ell}+q y_{k-\ell}= & \frac{d}{d t}\left(z_{\ell+1}+q \mathcal{L}_{\ell}\left(Z_{\ell}\right)\right) \\
& +q\left(z_{\ell+1}+q \mathcal{L}_{\ell}\left(Z_{\ell}\right)\right) \\
= & z_{\ell+2}+q \sum_{p=1}^{\ell} \frac{\partial \mathcal{L}_{\ell}}{\partial z_{p}}\left(Z_{\ell}\right) z_{p+1} \\
& +q\left(z_{\ell+1}+q \mathcal{L}_{\ell}\left(Z_{\ell}\right)\right) \\
= & z_{\ell+2}+q \mathcal{L}_{\ell+1}\left(Z_{\ell+1}\right),
\end{aligned}
$$

which allows us to solve for $\mathcal{L}_{\ell+1}$ and $y_{k-\ell-1}$. Since $z_{1}=$ $y_{k}$, we can also use (A.2) to solve for $z$ in terms of $y$, so this provides $P$. Also, by differentiating both sides of (A.2) with the choice $i=k-1$, we obtain

$$
\dot{y}_{1}=u+h(t, x, z)+q \sum_{p=1}^{k-1} \frac{\partial \mathcal{L}_{k-1}}{\partial z_{p}}\left(Z_{k-1}\right) z_{p+1},
$$

so $\dot{y}_{1}(t)=-q y_{1}(t)+m(x(t), y(t))+u(t)$ holds by choosing

$$
\begin{array}{r}
\left.m(x, y)=h(t, x, z)+q \sum_{\begin{array}{c}
p=1 \\
+q
\end{array}}^{k-1} \frac{\partial \mathcal{L}_{k-1}}{\partial z_{p}}\left(Z_{k-1}\right) z_{p+1}+q \mathcal{L}_{k-1}\left(Z_{k-1}\right)\right) .
\end{array}
$$

This proves the lemma.

\section{REFERENCES}

[1] N. Bekiaris-Liberis and M. Krstic. Compensation of state-dependent input delay for nonlinear systems. IEEE Trans. Automat. Control, 58(2):275-289, 2013.

[2] N. Bekiaris-Liberis and M. Krstic. Nonlinear Control Under Nonconstant Delays. SIAM, Philadelphia, 2013.

[3] R. Freeman and L. Praly. Integrator backstepping for bounded controls and control rates. IEEE Trans. Automat. Control, 43(2):258-262, 1998.

[4] A. Gruszka, M. Malisoff, and F. Mazenc. Tracking control and robustness analysis for PVTOL aircraft under bounded feedbacks. International J. Robust Nonlinear Control, 22(17):1899-1920, 2012.

[5] A. Isidori. Nonlinear Control Systems, Third Edition. Springer, New York, 1995.

[6] Z-P. Jiang and H. Nijmeijer. A recursive technique for tracking control of nonholonomic systems in chained form. IEEE Trans. Automat. Control, 44(2):265-279, 1999.

[7] H. Khalil. Nonlinear Systems, Third Edition. Prentice Hall, Englewood Cliffs, NJ, 2002.

[8] M. Krstic. Delay Compensation for Nonlinear, Adaptive, and PDE Systems. Birkhauser, Boston, 2009.

[9] M. Malisoff and F. Mazenc. Constructions of Strict Lyapunov Functions. Springer, New York, 2009.

[10] Mathematica. The world's definitive system for modern technical computing. http://www.wolfram.com/mathematica/, 2015.

[11] F. Mazenc and S. Bowong. Backstepping with bounded feedbacks for time-varying systems. SIAM J. Control Optimization, 43(3):856-871, 2004.

[12] F. Mazenc and A. Iggidr. Backstepping with bounded feedbacks. Systems and Control Letters, 51(3-4):235-245, 2004.

[13] F. Mazenc and M. Malisoff. Bounded backstepping approach under input delays. In Proceedings of the 2015 European Control Conference, Linz, Austria, 2015, pp. 2056-2061.

[14] F. Mazenc and M. Malisoff. New control design for bounded backstepping under input delays. Automatica, 66:48-55, 2016.

[15] F. Mazenc, M. Malisoff, and Z. Lin. Further results on input-to-state stability for nonlinear systems with delayed feedbacks. Automatica, 44(9):2415-2421, 2008.

[16] F. Mazenc, S. Mondie, and S-I. Niculescu. Global asymptotic stabilization for chains of integrators with a delay in the input. IEEE Trans. Automat. Control, 48(1):57-63, 2003.

[17] F. Mazenc, S-I. Niculescu, and M. Bekaik. Backstepping for nonlinear systems with delay in the input revisited. SIAM J. Control Optimization, 49(6):2263-2278, 2011.

[18] F. Mazenc and L. Praly. Adding an integration and global asymptotic stabilization of feedforward systems. IEEE Trans. Automat. Control, 41(11):1559-1578, 1996.

[19] R. Olfati-Saber. Nonlinear Control of Underactuated Mechanical Systems with Application to Robotics and Aerospace Vehicles. PhD Dissertation, Department of Electrical Engineering and Computer Science, Massachusetts Institute of Technology, 2001.

[20] R. Olfati-Saber. Global configuration stabilization for the VTOL aircraft with strong input coupling. IEEE Trans. Automat. Control, 47(11):1949-1952, 2002.

[21] A. Teel. Global stabilization and restricted tracking for multiple integrators with bounded controls. Systems and Control Letters, 18:165171, 1992.

[22] J. Tsinias. Input to state stability properties of nonlinear systems and applications to bounded feedback stabilization using saturation. ESAIM Control, Optimization, and Calculus of Variations, 2:57-87, 1997.

[23] J. Tsinias. Backstepping design for time-varying systems and application to partial-static feedback and asymptotic tracking. Systems and Control Letters, 39:219-227, 2000.

[24] J. Tsinias and I. Karafyllis. ISS property for time-varying systems and application to partial-static feedback stabilization and asymptotic tracking. IEEE Trans. Automat. Control, 44(11):2179-2184, 1999. 